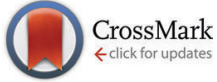

Cite this: Phys. Chem. Chem. Phys., $2015,17,31434$

Received 20th January 2015, Accepted 31st March 2015

DOI: $10.1039 / c 5 c p 00352 k$

www.rsc.org/pccp

\section{Configuration interaction singles based on the real-space numerical grid method: Kohn-Sham versus Hartree-Fock orbitals $\dagger$}

\author{
Jaewook Kim $\ddagger$ Kwangwoo Hong, $\ddagger$ Sunghwan Choi, Sang-Yeon Hwang and \\ Woo Youn Kim*
}

\begin{abstract}
We developed a program code of configuration interaction singles (CIS) based on a numerical grid method. We used Kohn-Sham (KS) as well as Hartree-Fock (HF) orbitals as a reference configuration and Lagrange-sinc functions as a basis set. Our calculations show that KS-CIS is more cost-effective and more accurate than HF-CIS. The former is due to the fact that the non-local HF exchange potential greatly reduces the sparsity of the Hamiltonian matrix in grid-based methods. The latter is because the energy gaps between KS occupied and virtual orbitals are already closer to vertical excitation energies and thus KS-CIS needs small corrections, whereas HF results in much larger energy gaps and more diffuse virtual orbitals. KS-CIS using the Lagrange-sinc basis set also shows a better or a similar accuracy to smaller orbital space compared to the standard HF-CIS using Gaussian basis sets. In particular, KS orbitals from an exact exchange potential by the Krieger-Li-lafrate approximation lead to more accurate excitation energies than those from conventional (semi-) local exchange-correlation potentials.
\end{abstract}

\section{Introduction}

Rapidly growing interest in computational materials design ${ }^{1-5}$ and quantum biology ${ }^{6}$ has encouraged development of innovative methods for accurate electronic structure calculations of large systems. $^{7-12}$ Though quantum chemistry using atom-centred basis functions such as Gaussian basis sets shows unrivalled performance for small and medium size molecules in terms of computation costs, they face the limit of their capabilities in dealing with large systems. Apparently, grid-based methods are promising alternatives, because their computational costs can be reduced through massive parallelization as well as linear scaling algorithms $^{7-10,13-15}$ and their accuracy can be systematically improved by single parameter control without optimizing systemor method-dependent parameters.

Most available codes using numerical grid basis sets adopt density functional theory (DFT) for ground state calculations and time-dependent DFT for excited state calculations..$^{7-10,16}$ Though (time-dependent) DFT offers a cost-effective way to describe large systems with reliable accuracy in many cases, it

Department of Chemistry, KAIST, 291 Daehak-ro, Yuseong-gu, Daejeon 305-701,

Korea. E-mail: wooyoun@kaist.ac.kr

$\dagger$ Electronic supplementary information (ESI) available: KLI virtual orbitals of formaldehyde; CIS excitation energies and CIS coefficients for three valence excited states of formaldehyde, benzene, formamide, and hydrogen molecules. See DOI: $10.1039 / \mathrm{c} 5 \mathrm{cp} 00352 \mathrm{k}$

\$ Jaewook Kim and Kwangwoo Hong contributed equally to this work. fails even qualitatively for strongly-correlated systems, ${ }^{17,18}$ since it relies on a single-determinant approach.

Strong correlation effects of electrons can readily be captured by using multiconfiguration (MC) methods. To our best knowledge, however, no traditional wave function theory has been used in realspace numerical grid methods. The so-called post-Hartree-Fock (post-HF) approaches may not be adequate for numerical grid basis sets, since the nonlocal HF exchange operator greatly reduces the sparsity of the Hamiltonian matrix and consequently increases computational costs. The optimized effective potential (OEP) method which constructs a local potential from the nonlocal HF exchange energy can be employed to circumvent such a numerical burden. Then, one is able to use the framework of a conventional Kohn-Sham (KS) DFT method but that a local exchange potential is replaced by an orbital-dependent exact exchange (EXX) potential. For practical purposes, the Krieger-Li-Iafrate (KLI) approximation, ${ }^{19-23}$ the localized HF method, ${ }^{24-26}$ and the common energy denominator approximation ${ }^{27,28}$ have been developed to implement EXX in a cost-effective manner.

MC methods require virtual orbitals in addition to occupied orbitals. KS orbitals obtained from EXX may show similar or better performance for application to MC methods than HF orbitals. In our previous work, we investigated the features of KLI orbitals through comparison to HF orbitals and conventional KS orbitals obtained from local density approximation (LDA) and generalized gradient approximation (GGA). ${ }^{29}$ Since the KLI potential has been derived from the HF exchange term, 
occupied orbitals of KLI are similar to those of HF in terms of energy and shape. However, their virtual orbitals have no such similarity. KLI virtual orbitals are the result of an $n$-electron system as usual KS orbitals and thus their energies are relatively closer to vertical excitation energies. ${ }^{29-31}$ In contrast, HF yields virtual orbitals of an $(n+1)$-electron system and thus their energies are regarded as electron affinities. In addition, the KLI potential has the correct $-1 / r$ asymptotic behaviour, while LDA and GGA potentials exponentially decay due to self-interaction errors. ${ }^{29,32}$ As a result, the KLI potential well describes Rydberg-like states with a number of bound virtual orbitals, whereas HF or conventional KS orbitals have few bound states. ${ }^{29}$ In fact, KLI orbitals are relatively closer to the so-called exact KS orbitals. ${ }^{29-31}$ Therefore, we expect that these distinct features of KLI orbitals certainly offer benefits for excited state calculations using MC methods.

There have been preceding research studies on MC methods using KS orbitals. For instance, the accurate excitation energies of atoms and small molecules could be obtained using (multireference) configuration interaction (CI) methods. ${ }^{33-39}$ Gutlé et al. reported that a coupled-cluster singles and doubles method using approximated OEP orbitals produced quantitatively similar correlation energies for atoms compared to the HF version. ${ }^{40} \mathrm{CI}$ with configurations obtained from constrained DFT described various excited state properties of molecules in a qualitatively correct manner, where KS theory fails. ${ }^{41}$

It should be noted that the aforementioned KS-MC methods are based on atom-centred basis sets. Yanai et al. devised the linear response TD-HF method using multiresolution multiwavelet basis sets. ${ }^{42}$ However, the extension of such an approach to double or higher excitations will be an intractable task due to the increased dimensionality of response function accordingly. In this work, we, for the first time, developed a CI method based on real-space numerical grids using the traditional matrix approach, which can be straightforwardly extended to higher levels. For comparison, we used KLI, GGA, and HF orbitals to build a reference configuration. Specifically, the CI code includes only single excitations as a prototype and was implemented in our KS-DFT code that uses Lagrange-sinc functions (LSFs) as a basis set. We already reported the details of our KS-DFT code and its accuracy for ground state calculations of molecules in comparison to the results of widelyused Gaussian basis sets. ${ }^{29,43}$ Here, we briefly explain the features of the LSFs as a basis set and the KLI approximation, and then describe details of the CIS implementation using LSFs. We compared between KLI-, GGA-, and HF-CIS results for excited state calculations of molecules and finally draw conclusions with future outlook on the extension of the present CI method to double and higher excitations.

\section{Method}

\section{Lagrange-sinc functions as a basis set}

A sinc function localized on a grid point $x_{i}($ e.g., the red curve in Fig. 1) is given by

$$
L_{i}^{\operatorname{sinc}}(x)=\frac{1}{\sqrt{h}} \frac{\sin \left[\pi\left(x-x_{i}\right) / h\right]}{\pi\left(x-x_{i}\right) / h},
$$

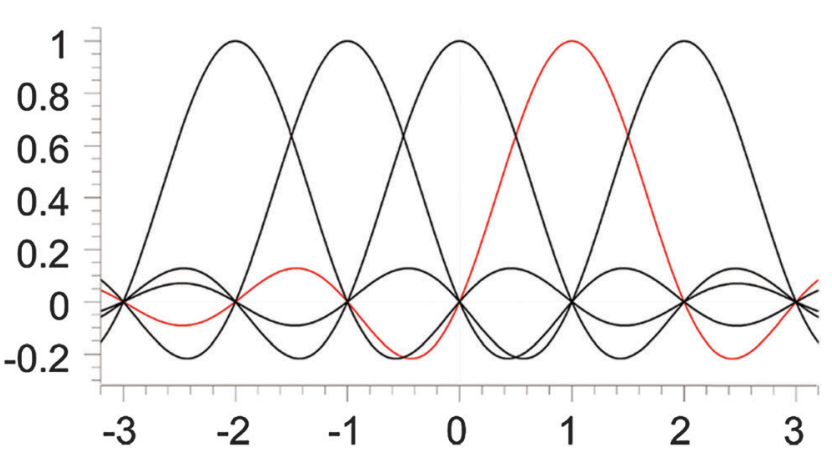

Fig. 1 Lagrange-sinc functions.

where $h$ is a scaling factor. The roots of a sinc function form a set of grid points with uniform spacing $h$. Sinc functions localized on those grid points have the following intriguing attributes: cardinality and orthonormality written as

$$
L_{i}^{\mathrm{sinc}}\left(x_{j}\right)=\delta_{i j} \text { and } \int L_{i}^{\mathrm{sinc}}(x) L_{j}^{\mathrm{sinc}}(x) \mathrm{d} x=\delta_{i j},
$$

respectively. In fact, there is a family of functions, namely the Lagrange functions that share the above two attributes. Therefore, sinc functions localized on grid points with uniform spacing $h$ are called the Lagrange-sinc functions (LSFs), while the grid is named as the Lagrange-sinc mesh. For more details of the Lagrange functions, we refer to ref. 44 and 45 .

The LSFs can be used as a basis set to represent HF or KS orbitals;

$$
\phi_{n}(\mathbf{r})=\sum_{i, j, k} c_{i j k}^{n} L_{i j k}^{\operatorname{sinc}}(x, y, z),
$$

where $c_{i j k}^{n}$ is the expansion coefficient of a three-dimensional LSF defined by

$$
L_{i j k}^{\operatorname{sinc}}(x, y, z) \equiv L_{i}^{\text {sinc }}(x) L_{j}^{\operatorname{sinc}}(y) L_{k}^{\text {sinc }}(z) .
$$

Then, a matrix element of any one-electron operator can be obtained as

$$
\begin{aligned}
A_{i j k i^{\prime} j^{\prime} k^{\prime}} & =\left\langle L_{i j k}^{\operatorname{sinc}}|\hat{A}| L_{i^{\prime} j^{\prime} k^{\prime}}^{\operatorname{sinc}}\right\rangle \\
& =\sum_{l, m, n} L_{i j k}^{\operatorname{sinc}}\left(x_{l}, y_{m}, z_{n}\right) \hat{A}\left(x_{l}, y_{m}, z_{n}\right) L_{i^{\prime} j^{\prime} k^{\prime}}^{\operatorname{sinc}}\left(x_{l}, y_{m}, z_{n}\right) .
\end{aligned}
$$

Due to the cardinality of LSFs, the matrix of any local potential $v(\mathbf{r})$ has non-zero values only in its diagonal elements;

$$
\begin{aligned}
v_{i j k i^{\prime} j^{\prime} k^{\prime}} & =\sum_{l, m, n} L_{i j k}^{\mathrm{sinc}}\left(x_{l}, y_{m}, z_{n}\right) v\left(x_{l}, y_{m}, z_{n}\right) L_{i^{\prime} j^{\prime} k^{\prime}}^{\mathrm{sinc}}\left(x_{l}, y_{m}, z_{n}\right) \\
& =v\left(x_{i}, y_{j}, z_{k}\right) \delta_{i i^{\prime}} \delta_{j j^{\prime}} \delta_{k k^{\prime}} .
\end{aligned}
$$

Since LSFs are analytically differentiable, the matrix elements of the kinetic energy operator can also be obtained analytically;

$$
\begin{aligned}
T_{i j k i^{\prime} j^{\prime} k^{\prime}} & =\left\langle L_{i j k}^{\operatorname{sinc}}\left|-\frac{1}{2} \nabla^{2}\right| L_{i^{\prime} j^{\prime} k^{\prime}}^{\operatorname{sinc}}\right\rangle \\
& =T_{i i^{\prime}} \delta_{j j^{\prime}} \delta_{k k^{\prime}}+\delta_{i i^{\prime}} T_{j j^{\prime}} \delta_{k k^{\prime}}+\delta_{i i^{\prime}} \delta_{j j^{\prime}} T_{k k^{\prime}},
\end{aligned}
$$


where for the $x$-direction as an example, we have

$$
T_{i=i^{\prime}}=\frac{1}{h^{2}} \frac{\pi^{2}}{6} \text { and } T_{i \neq i^{\prime}}=\frac{(-1)^{i-i^{\prime}}}{h^{2}} \frac{1}{\left(x_{i}-x_{i^{\prime}}\right)^{2}} .
$$

It should be noted that the overlap matrix between LSFs is an identity matrix due to the orthonormality of LSFs.

LSFs apparently have beneficial features for application in electronic structure calculations. Compared to the finite difference method which shares the same uniform grid, LSFs guarantee more accurate kinetic energy thanks to the analytic expression for derivatives. However, the use of pseudopotentials is essential for cost-effective calculations. We have implemented a KS-DFT code using LSFs as a basis set with norm-conserving pseudopotentials. ${ }^{43}$ Our previous work showed that accuracy of the Lagrange-sinc basis set can be systematically improved for atomization energies, ionization energies, electron affinities, and polarizabilities of atoms and molecules. In particular, we demonstrated that it is suitable to describe highly diffuse and polarized orbitals. For more details of implementation and its accuracy, we refer to ref. 43.

\section{Krieger-Li-Iafrate approximation}

The KLI approximation has been regarded as a practical approach to obtain a local EXX potential. We implemented this method in our code and addressed the distinct features of KLI orbitals compared to HF or conventional LDA/GGA orbitals. ${ }^{29}$ Here, we provide a brief review on the KLI approximation but for more details we refer to ref. 23 and 29.

The HF exchange energy, $E_{x}^{\mathrm{HF}}$, can be written as

$$
\begin{aligned}
E_{x}^{\mathrm{HF}}\left[\left\{\phi_{i \sigma}\right\}\right] & =\sum_{\sigma} \sum_{i=1}^{N_{\sigma}}\left\langle\phi_{i \sigma}\left|\hat{v}_{x, i \sigma}^{\mathrm{HF}}\left[\left\{\phi_{i \sigma}\right\}\right]\right| \phi_{i \sigma}\right\rangle \\
& =-\frac{1}{2} \sum_{\sigma} \sum_{i, j=1}^{N_{\sigma}} \iint \mathrm{d} \mathbf{r} \mathrm{d} \mathbf{r}^{\prime} \frac{\phi_{i \sigma}{ }^{*}(\mathbf{r}) \phi_{j \sigma}{ }^{*}\left(\mathbf{r}^{\prime}\right) \phi_{j \sigma}(\mathbf{r}) \phi_{i \sigma}\left(\mathbf{r}^{\prime}\right)}{\left|\mathbf{r}-\mathbf{r}^{\prime}\right|},
\end{aligned}
$$

where $N_{\sigma}$ and $\left\{\phi_{i \sigma}\right\}$ are the number of electrons with spin $\sigma$ and HF or KS orbitals, respectively. $\hat{v}_{x, i \sigma}^{\mathrm{HF}}$ is the HF exchange potential operator, which can be formulated by rearranging eqn (1) as follows:

$$
\begin{aligned}
v_{x, i \sigma}^{\mathrm{HF}}\left[\left\{\phi_{i \sigma}\right\}\right](\mathbf{r}) & =\frac{1}{\phi_{i \sigma}{ }^{*}(\mathbf{r})} \frac{\delta E_{x}^{\mathrm{HF}}\left[\left\{\phi_{i \sigma}\right\}\right]}{\delta \phi_{i \sigma}(\mathbf{r})} \\
& =-\frac{1}{\phi_{i \sigma}{ }^{*}(\mathbf{r})} \sum_{j=1}^{N_{\sigma}} \phi_{j \sigma}^{*}(\mathbf{r}) \int \mathrm{d} \mathbf{r}^{\prime} \frac{\phi_{i \sigma}^{*}\left(\mathbf{r}^{\prime}\right) \phi_{j \sigma}\left(\mathbf{r}^{\prime}\right)}{\left|\mathbf{r}-\mathbf{r}^{\prime}\right|}
\end{aligned}
$$

Using the HF exchange potential given in eqn (2), the KLI potential can be represented as

$$
v_{x, \sigma}^{\mathrm{KLI}}(\mathbf{r})=\frac{1}{\rho_{\sigma}(\mathbf{r})} \sum_{i}^{N_{\sigma}}\left|\phi_{i \sigma}(\mathbf{r})\right|^{2}\left(v_{x, i \sigma}^{\mathrm{HF}}(\mathbf{r})-\left(\bar{v}_{x, i \sigma}^{\mathrm{HF}}-\bar{v}_{x, i \sigma}^{\mathrm{KLI}}\right)\right),
$$

where $\bar{v}_{x, i \sigma}^{\mathrm{HF}} \equiv\left\langle\phi_{i \sigma}\left|\hat{v}_{x, i \sigma}^{\mathrm{HF}}\right| \phi_{i \sigma}\right\rangle$ and $\bar{v}_{x, i \sigma}^{\mathrm{KLI}} \equiv\left\langle\phi_{i \sigma}\left|\hat{v}_{x, i \sigma}^{\mathrm{KLI}}\right| \phi_{i \sigma}\right\rangle$. It should be noted that unlike the original OEP integral equation, the KLI potential can be obtained using simple linear algebra with only occupied orbitals. ${ }^{19,20}$

\section{CIS based on Lagrange-sinc functions}

We implemented a CIS method using KS orbitals in our code. We use a Slater determinant composed of ground state KS orbitals, $\left|\Phi_{0}\right\rangle$, as a reference configuration. Then, we performed CIS calculations almost in the same manner by the traditional HF-CIS method. For instance, a single excitation configuration $\left|\Phi_{i}^{a}\right\rangle$ can be generated by promoting one electron from an occupied orbital $\phi_{i}$ to a virtual orbital $\phi_{a}$. Because we use numerical grid methods and pseudopotentials, the generation of single excitation configurations should be carried out within an active orbital space which includes only valence orbitals in the occupied space and a finite number of virtual orbitals truncated by an energy threshold. It should also be noted that KS orbitals are not the eigenfunctions of the Fock operator and thus unlike the HF-CI method, Brillouin's theorem does not hold for namely the KS-CI method as shown in eqn (3).

$$
\begin{aligned}
\left\langle\Phi_{0}|\hat{H}| \Phi_{i}^{a}\right\rangle & =\langle i|\hat{h}| a\rangle+\sum_{k}\langle i k \| a k\rangle \\
& =\left\langle i\left|\hat{H}^{\mathrm{KS}}\right| a\right\rangle+\left\langle i\left|\hat{v}_{x}^{\mathrm{HF}}-\hat{v}_{x}^{\mathrm{KS}}\right| a\right\rangle \\
& =\left\langle i\left|\hat{v}_{x}^{\mathrm{HF}}-\hat{v}_{x}^{\mathrm{KS}}\right| a\right\rangle \neq 0 .
\end{aligned}
$$

To consider the spin state of each excitation, we used a configuration state function $\left|{ }^{2 S+1} \Phi_{i}^{a}\right\rangle$, where $2 S+1$ indicates the spin multiplicity. A KS-CIS matrix element for a singlet excitation can be written as

$$
\begin{aligned}
\left\langle{ }^{1} \Phi_{i}^{a}\left|\hat{H}-E_{0}\right|^{1} \Phi_{j}^{b}\right\rangle= & \left(\varepsilon_{a}-\varepsilon_{i}\right) \delta_{a b} \delta_{i j}+2(a i \mid j b)-(a b \mid j i) \\
& +\left\langle a\left|\hat{v}_{x}^{\mathrm{HF}}-\hat{v}_{x}^{\mathrm{KS}}\right| b\right\rangle \delta_{i j}-\left\langle j\left|\hat{v}_{x}^{\mathrm{HF}}-\hat{v}_{x}^{\mathrm{KS}}\right| i\right\rangle \delta_{a b},
\end{aligned}
$$

where $\hat{H}, E_{0}$ and $\varepsilon_{i / a}$ denote a molecular Hamiltonian under the Born-Oppenheimer approximation, the corresponding ground state total energy, and the $i / a$-th orbital energy, respectively. In the process of constructing the KS-CIS matrix, the evaluation of the following two-centre integral is the most time-consuming part.

$$
(i j \mid k l)=\int \mathrm{d} \mathbf{r}_{1} \int \mathrm{d} \mathbf{r}_{2} \frac{\phi_{i}^{*}\left(\mathbf{r}_{1}\right) \phi_{j}\left(\mathbf{r}_{1}\right) \phi_{k}^{*}\left(\mathbf{r}_{2}\right) \phi_{l}\left(\mathbf{r}_{2}\right)}{\left|\mathbf{r}_{1}-\mathbf{r}_{2}\right|}
$$

To efficiently evaluate the integral in eqn (5), we employed the interpolating scaling function method which has been originally used to compute Hartree potentials. ${ }^{4-51}$ First, eqn (5) can be rewritten as

$$
(i j \mid k l)=\int \mathrm{d} \mathbf{r}_{1} \phi_{i}^{*}\left(\mathbf{r}_{1}\right) \phi_{j}\left(\mathbf{r}_{1}\right) K_{k l}\left(\mathbf{r}_{1}\right),
$$

where we define

$$
\begin{aligned}
K_{k l}\left(\mathbf{r}_{1}\right) & \equiv \int \mathrm{d} \mathbf{r}_{2} \frac{\phi_{k}^{*}\left(\mathbf{r}_{2}\right) \phi_{l}\left(\mathbf{r}_{2}\right)}{\left|\mathbf{r}_{1}-\mathbf{r}_{2}\right|} \\
& =\frac{2}{\sqrt{\pi}} \int_{0}^{\infty} \mathrm{d} t \int \mathrm{d} \mathbf{r}_{2} \mathrm{e}^{-t^{2}\left(r_{1}-r_{2}\right)^{2}} \rho_{k l}\left(\mathbf{r}_{2}\right),
\end{aligned}
$$

and $\rho_{k l}(\mathbf{r}) \equiv \phi_{k}^{*}(\mathbf{r}) \phi_{l}(\mathbf{r})$. In eqn (7), $\left|\mathbf{r}_{1}-\mathbf{r}_{2}\right|^{-1}$ was replaced by the integration of a Gaussian function. Then, we expand $\rho_{k l}(\mathbf{r})$ with LSFs as 


$$
\rho_{k l}(\mathbf{r}) \approx \sum_{a b c} c_{a b c}^{k l} L_{a b c}^{\mathrm{sinc}}(x, y, z)
$$

and insert it into eqn (7). Using the fact that both the Gaussian function and LSFs can be written as the products of three functions, eqn (7) can be re-expressed as

$$
\begin{aligned}
K_{k l}\left(\mathbf{r}_{1}\right)= & \frac{2}{\sqrt{\pi}} \sum_{s} w_{s} \sum_{a b c} c_{a b c}^{k l}\left[\int_{-\infty}^{\infty} \mathrm{e}^{-t_{s}{ }^{2}\left(x_{1}-x_{2}\right)^{2}} L_{a}^{\text {sinc }}\left(x_{2}\right) \mathrm{d} x_{2}\right. \\
& \left.\times \int_{-\infty}^{\infty} \mathrm{e}^{-t_{s}^{2}\left(y_{1}-y_{2}\right)^{2}} L_{b}^{\text {sinc }}\left(y_{2}\right) \mathrm{d} y_{2} \int_{-\infty}^{\infty} \mathrm{e}^{-t_{s}{ }^{2}\left(z_{1}-z_{2}\right)^{2}} L_{c}^{\text {sinc }}\left(z_{2}\right) \mathrm{d} z_{2}\right] \\
& +\frac{\pi}{t_{f}^{2}} \rho_{k l}\left(\mathbf{r}_{1}\right)
\end{aligned}
$$

which just requires to perform three one-dimensional integrals. The integral along $t_{s}$ can be evaluated using a Gaussian quadrature method with 51 grid points $\left\{t_{s}\right\}$ and corresponding weight factors $\left\{w_{s}\right\}$, and $t_{f}$ is the largest value in $\left\{t_{s}\right\}$. Since we eventually need to compute eqn (6) on a given Lagrange-mesh, eqn (9) can be transformed into a matrix form as

$$
K_{k l}\left(\mathbf{r}^{\prime}\right)=\frac{2}{\sqrt{\pi}} \sum_{s} w_{s} \sum_{a} F_{a^{\prime} a}^{x, s} \sum_{b} F_{b^{\prime} b}^{y, s} \sum_{c} F_{c^{\prime} c}^{z, s} c_{a b c}^{k l}+\frac{\pi}{t_{f}^{2}} \rho_{k l}\left(\mathbf{r}^{\prime}\right),
$$

where a so-called $F$ matrix is given by

$$
F_{a^{\prime} a}^{x, s}=\int_{-\infty}^{\infty} \mathrm{e}^{-t_{\mathrm{s}}^{2}\left(x_{a^{\prime}}-x_{2}\right)^{2}} L_{a}^{\text {sinc }}\left(x_{2}\right) \mathrm{d} x_{2} .
$$

Eqn (11) is independent of orbitals and therefore calculated once at the beginning of calculations. The computational cost of the summation in eqn (10) is proportional to $N_{\text {grid }}^{4 / 3}$ where $N_{\text {grid }}$ is the total number of grid points on the Lagrange-mesh. ${ }^{49}$ Consequently the cost of calculating $\left\{K_{k l}\right\}$ for all orbital pairs of CIS is scaled as $N_{\text {grid }}^{4 / 3}\left(N_{\text {occ }}+N_{\text {vir }}\right)^{2}$ where $N_{\text {occ }}$ and $N_{\text {vir }}$ are the numbers of occupied and virtual orbitals in an active space, respectively. Finally, eqn (6) is computed using a Gaussian quadrature method on the Lagrange-mesh. Considering all orbital pairs in the active space, the cost of computing eqn (6) using pre-determined $K_{k l}$ is proportional to $N_{\text {grid }} \cdot N_{\text {occ }}{ }^{2} N_{\text {vir }}{ }^{2}$. As a result, the total computational cost of the two-centre integrals for CIS is scaled as $N_{\text {grid }}^{4 / 3}\left(N_{\text {occ }}+N_{\text {vir }}\right)^{2}+N_{\text {grid }} \cdot N_{\text {occ }}{ }^{2} N_{\text {vir }}{ }^{2}$. If we use KLI orbitals as a reference configuration, the part of $\left\{K_{k l}\right\}$ corresponding to occupied orbitals is already computed during ground state calculations [eqn (2)] and thus additional costs for CIS calculations will be $N_{\text {grid }}^{4 / 3}\left(N_{\text {vir }}^{2}+N_{\text {occ }} N_{\text {vir }}\right)+N_{\text {grid }} \cdot N_{\text {occ }}^{2} N_{\text {vir }}^{2}$.

\section{Calculation details}

We performed a series of CIS calculations to obtain the excitation energies of $\mathrm{H}_{2}$, formaldehyde, formamide, and benzene. The bond length of $\mathrm{H}_{2}$ was set to $1.5 \mathrm{Bohr}$, while the geometries of the other molecules were obtained from ref. 52 and 53. For comparison, we used both KS-CIS and HF-CIS methods with various basis sets. KS orbitals for CIS calculations have been calculated using the KLI exchange-only potential and the $\mathrm{PBE}^{54}$ exchange-correlation potential. Because virtual orbitals are more diffuse than occupied orbitals, their energies and shapes would be more sensitive to the choice of basis set than those of occupied orbitals. Therefore, we studied the effects of simulation box size and grid spacing on CIS results. Also, we investigated the dependence of the CIS results on active space size that is denoted by the notation, (n-electrons, m-orbitals), used in conventional post-HF methods.

We used our KS-DFT code to obtain KLI and PBE orbitals. To perform HF-CIS calculations using a numerical grid basis set, we first obtained HF orbitals from the Octopus program, ${ }^{7}$ which uses the finite difference method, and then put them in our code for the remaining calculations. We applied an identical grid setting with a sphere-shape simulation box and identical norm-conserving pseudopotentials at the PBE level ${ }^{55}$ to obtain KLI, PBE, and HF orbitals. We also carried out HF-CIS calculations using (aug)-cc-pVNZ ( $=\mathrm{D}, \mathrm{Q})$ basis sets as implemented in the Gaussian 09 package $^{56}$ and compared the results with those of the grid basis sets. All orbital figures were drawn with the isovalue of 0.014 .

\section{Results}

\section{Energy convergence}

We first investigated the convergence of CIS excitation energies as a function of the radius of a spherical simulation box and the scaling factor $h$. As a test set, we used the three valence excitation energies of formaldehyde $\left(1^{1} \mathrm{~A}_{2}, 1^{1} \mathrm{~B}_{2}\right.$, and $\left.1^{1} \mathrm{~B}_{1}\right)$ obtained from KLI-CIS with the active space $(12,24)$.

Simulation box size. The CIS calculations were carried out using a spherical simulation box with a fixed scaling factor ( $h=0.3$ Bohr), as shown in Fig. 2(a). The reference values for each excitation energy were obtained using the radius of 21.0 Bohr. The energies of $1^{1} \mathrm{~A}_{2}$ and $1^{1} \mathrm{~B}_{1}$ readily converge with a small deviation $(<0.02 \mathrm{eV})$ over the whole range, but the energy of $1^{1} \mathrm{~B}_{2}$ converges as the radius is larger than 13.5 Bohr. $1^{1} \mathrm{~A}_{2}$ and $1^{1} \mathrm{~B}_{1}$ correspond to the excitations from $2 b_{2}$ and $5 a_{1}$, respectively, to the $\pi^{*}$ orbital $\left(2 b_{1}\right)$, while $1^{1} B_{2}$ is due to the excitation from $2 b_{2}$ to the $\sigma^{*}$ orbital $\left(6 \mathrm{a}_{1}\right)$. The $\sigma^{*}$ orbital is more diffuse than the $\pi^{*}$

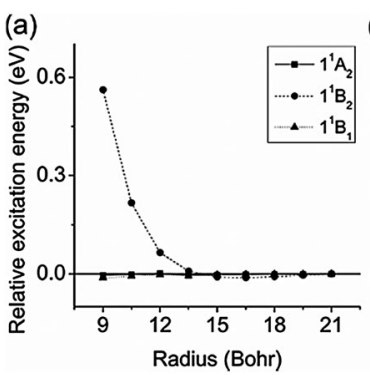

(b)

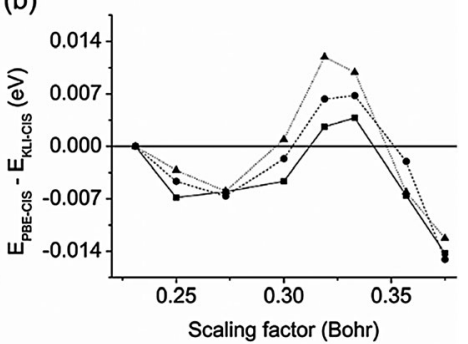

Fig. 2 (a) The convergence of the three valence excitation energies of formaldehyde obtained from KLI-CIS with the active space $(12,24)$ as a function of the radius of a spherical simulation box. The reference values were obtained using radius $=21.0$ Bohr and $h=0.3$ Bohr. (b) The convergence of the difference between PBE-CIS and KLI-CIS excitation energies $\left(E_{\mathrm{PBE}-\mathrm{CIS}}-E_{\mathrm{KLI}-\mathrm{CIS}}\right)$ as a function of the scaling factor $h$. The reference values were obtained using $h=0.231$ Bohr. All values in (b) were calculated using radius $=15.0$ Bohr . 
orbital, and thus the former needs a relatively large simulation box to be converged (Fig. S1, ESI $\dagger$ ). (For the detailed assignments of each excitation, see Fig. S3, ESI. $\dagger$ ) In such a way, excitation energies involving diffuse orbitals strongly depend on the simulation box size, so that it must be carefully determined.

Scaling factor. The scaling factor is also an important parameter for energy convergence. Fig. 2(b) shows the convergence of excitation energy differences between PBE-CIS and KLI-CIS as a function of the scaling factor with a fixed radius of the simulation box (15.0 Bohr). The reference values of each excitation were obtained using a fine grid ( $h=0.231$ Bohr). The energy differences rapidly converge as $h$ decreases and when $h=0.3 \mathrm{Bohr}$, the maximum energy difference is about $0.007 \mathrm{eV}$. It should be noted that despite such rapid convergence of the energy differences, individual excitation energies themselves converge slowly because of the accuracy of the Gaussian quadrature integration for pseudopotentials on coarse grids (Fig. S2, ESI $\dagger$ ).

Hereafter, all calculations were done using a spherical simulation box with the radius of 15.0 Bohr and the scaling factor of 0.3 Bohr.

\section{Excitation energies from KLI-, PBE-, and HF-CIS}

We compared the excitation energies of formaldehyde, benzene, formamide, and hydrogen molecules obtained from KLI-, PBE-, and HF-CIS methods.

Determination of active space. We first need to determine an active space for CIS calculations. Apparently, the size of active space itself is a convergence parameter for excitation energies. The standard HF-CIS method uses whole orbital space as an active space, since computational costs of CIS are not significantly affected by the active space size. In the case of grid-based methods, the diagonalization of the Hamiltonian matrix typically produces millions of virtual orbitals. In practice, we need an appropriate threshold to restrict the virtual orbital space. The larger the active space, the more accurate the excitation energies. However, obtaining virtual orbitals from grid-based methods entails additional computational costs, because the matrix diagonalization is solved using iterative methods. Therefore, the active space size should be determined as a trade-off between accuracy and computational costs.

Fig. 3 shows the convergence of CIS excitation energies as a function of active space size with respect to the best known values computed by multireference methods (see the caption of Table 2). Fig. 3(a) shows that the absolute deviations of PBE- and KLI-CIS excitation energies for formaldehyde readily converge to certain values with small deviations $(<0.05 \mathrm{eV})$ as more than 30 virtual orbitals are included in the active space, whereas the HF-CIS results converge slowly (see Tables S1 and $\mathrm{S} 2$, ESI $\dagger$ for the complete data). We extended the convergence test of active space size for formaldehyde, benzene, formamide, and hydrogen molecules. In Fig. 3(b), the mean absolute deviation (MAD) values of PBE- and KLI-CIS converge rapidly, while that of HF-CIS converges slowly, implying that KS-CIS requires a smaller active space than HF-CIS for the same accuracy.

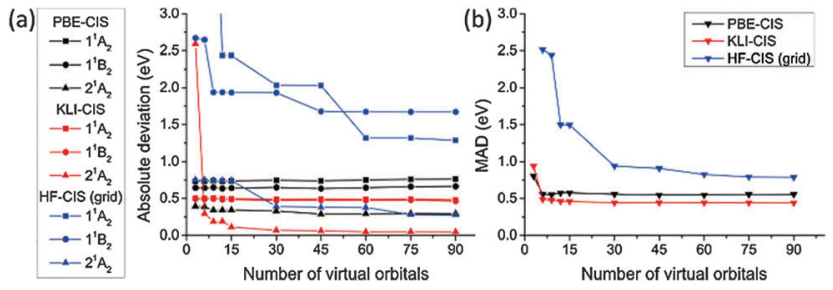

Fig. 3 (a) The convergence of the excitation energies of formaldehyde obtained from PBE-, KLI- and HF-CIS as a function of the number of virtual orbitals in the active space. (b) The convergence of the mean absolute deviation (MAD) of the three valence excitation energies for four molecules (see Table 2). The reference values were obtained from ref. 52 and 53 (see the caption of Table 2 ). All values were calculated using radius = 15.0 Bohr and scaling factor $h=0.3$ Bohr.

More intriguingly, the results of each method converge to different values. It is understandable by considering that the ground state determinants from each method are not orthogonal to each other and hence their CIS spaces span different subspaces of a given Hilbert space. Therefore, the accuracy of truncated CI methods should depend on the reference configuration obtained from ground state calculations. Fig. 3(b) demonstrates that KLI provides better reference configurations than PBE and HF.

From the convergence test in Fig. 3, we chose the active spaces shown in Table 1 for more detailed study on the individual excited states of each molecule in the following sections. In Table 1, the active space names, " 45 ", "6", and " 3 ", denote the number of virtual orbitals in the given active space. In the case of " 45 ”, the conventional expressions for the active spaces of formaldehyde, benzene, formamide, and hydrogen molecules correspond to $(12,51)$, $(30,60),(18,54)$, and $(2,46)$, respectively. For comparison, the active spaces for Gaussian basis sets are also given in Table 1.

Excitation energies. Table 2 summarizes the results of various CIS calculations. The second column indicates three valence excited states of each molecule and corresponding transition characteristics. The best estimation values in the third column were obtained using multireference methods (see the table caption for details). The following columns subsequently show the excitation energies of each state computed using HF-CIS (grid and Gaussian basis sets), KLI-, and PBE-CIS with various active spaces for the grid basis set or with various basis set sizes for the Gaussian case. Finally, the bottom row shows the MADs of each column with respect to the best estimation values. First, we compare the results using gridbased methods. The KLI-CIS results with the active space " 45 " have the smallest MAD $(0.44 \mathrm{eV})$. For the same active space, PBE-CIS gives a slightly larger MAD $(0.55 \mathrm{eV})$, but HF-CIS results in a significantly larger MAD $(0.91 \mathrm{eV})$.

To understand such differences, we invoke the features of KLI, PBE, and HF orbitals. As learned from our previous work, ${ }^{29}$ the energy gaps between occupied and virtual orbitals from KLI are relatively closer to vertical excitations. PBE orbital energies are overall upshifted compared to those of KLI, but their energy gaps are similar to those of KLI. However, HF has substantially large energy gaps. Furthermore, HF virtual orbitals are much 
Table 1 The active space of " 45 ", "6", "3", and Gaussian basis sets for various molecules ${ }^{a}$

\begin{tabular}{llllllll}
\hline & 45 & 6 & 3 & aug-cc-pVQZ & aug-cc-pVDZ & cc-pVQZ & cc-pVDZ \\
\hline Formaldehyde & $(12,51)$ & $(12,12)$ & $(12,9)$ & $(16,252)$ & $(16,64)$ & $(16,170)$ & $(16,38)$ \\
Benzene & $(30,60)$ & $(30,21)$ & $(30,18)$ & $(42,752)$ & $(42,192)$ & $(42,510)$ & $(42,114)$ \\
Formamide & $(18,54)$ & $(18,15)$ & $(18,12)$ & $(24,378)$ & $(24,96)$ & $(24,255)$ & $(24,57)$ \\
Hydrogen molecule & $(2,46)$ & $(2,7)$ & $(2,4)$ & $(2,92)$ & $(2,18)$ & $(2,60)$ & $(2,10)$
\end{tabular}

${ }^{a}$ The active space sizes are denoted by (n-electrons, m-orbitals).

Table 2 CIS excitation energies of formaldehyde, benzene, formamide, and hydrogen molecule (unit: eV)

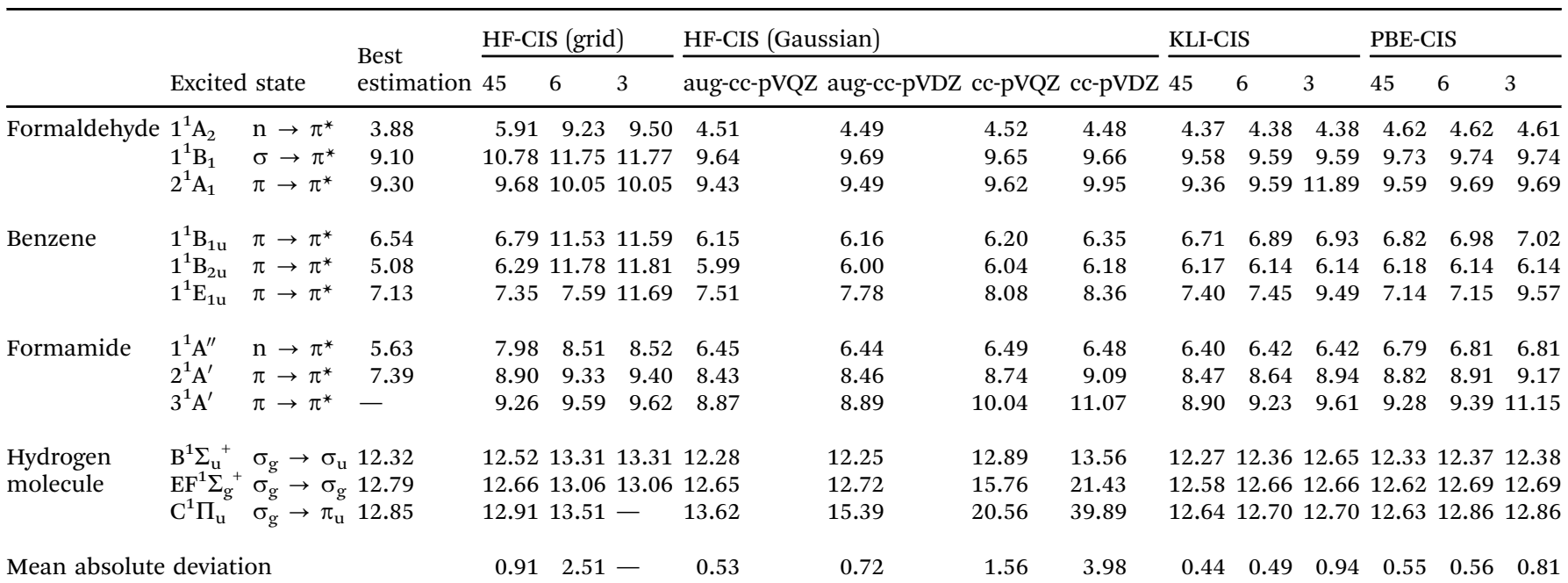

${ }^{a}$ The "Best estimation" means the best known values computed by multireference methods which were obtained from ref. 52 and 53 for benzene, formaldehyde, and formamide, and from ref. 57-59 for hydrogen molecule. " 45 ", "6", and " 3 " denote the size of active space used for each molecule (Table 1). For details, see the text. HF-CIS (Gaussian) means the HF-CIS results obtained using the Gaussian 09 package with Dunning basis sets. We note that all occupied and virtual orbitals of each molecule were used for CIS calculations using the Gaussian 09 package. Mean absolute deviations were calculated with respect to the "Best estimation" values.

more diffuse than KLI and PBE counterparts. As shown in eqn (4), CIS adds the energy correction from the two-centre integrals between single excitation configurations to the corresponding orbital energy gap. Therefore, KLI-CIS readily gives accurate excitation energies even with small correction from the two-centre integral, while HF-CIS needs large corrections. As a result, as long as we use the same size of active space, KLI-CIS will give us more accurate results than HF-CIS.

The comparison between aug-cc-pVDZ and cc-pVQZ manifests the importance of diffuse basis functions to obtain accurate excitation energies. For grid basis sets, the accuracy for diffuse states can be systematically tuned by controlling the size of the simulation box. ${ }^{43}$ If a sufficiently large simulation box is used, grid basis sets may perform better for diffuse states than Gaussian basis sets. In Table 2, however, the MADs of the grid-based HF-CIS are larger than those of aug-cc-pVNZ. This can be understood from the fact that the active space in the grid-based HF-CIS is much smaller than that in the Gaussian basis sets [cf. Fig. 3(b)].

We also found that the excitation energies from KS-CIS are relatively insensitive to the size of active space compared to HF-CIS. For example, the MAD of HF-CIS decreases by $1.60 \mathrm{eV}$ as the active space size increases from " 6 " to " 45 ", whereas that of KS-CIS decreases by $0.05 \mathrm{eV}$ for the same change. This is another important aspect for practical applications.
In order to further uncover the difference between KS-CIS and HF-CIS, we closely examine individual excitations of each molecule especially by focusing on their CIS coefficients and corresponding orbital shapes in the following section.

\section{CIS coefficients of individual excitations}

A singlet excited state wavefunction using CIS can be written as

$$
\left.\left|\Psi_{\mathrm{ex}}\right\rangle=c_{0}\left|\Phi_{0}\right\rangle+\left.\sum_{i, a} c_{i}^{a}\right|^{1} \Phi_{i}^{a}\right\rangle,
$$

where $c_{0}$ is zero in the case of HF-CIS by virtue of Brillouin's theorem (for Brillouin's theorem of KS-CIS, we refer to ref. 29). Due to the normalization condition of wavefunction, the coefficients satisfy the following relation.

$$
1=\left|c_{0}\right|^{2}+\sum_{i, a}\left|c_{i}^{a}\right|^{2}
$$

Thus, without loss of generality, we investigated the absolute squared values of CIS coefficients to analyse individual excitations instead of the coefficients themselves.

Formaldehyde. Though HF-CIS is generally inaccurate compared to KS-CIS, the HF-CIS excitation energy of formaldehyde to the first excited state, $1^{1} \mathrm{~A}_{2}$, is particularly worse; for the active space " 45 ", the excitation energy of HF-CIS is $\sim 1.5 \mathrm{eV}$ 


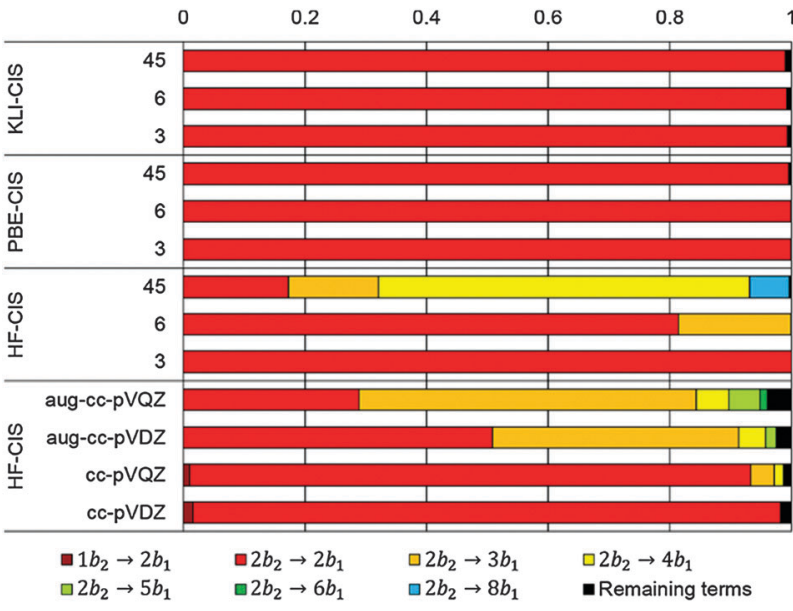

Fig. $4 \mathrm{CIS}$ coefficients for the $1^{1} \mathrm{~A}_{2}$ state of formaldehyde.

larger than that of KLI-CIS as shown in Table 2. Fig. 4 shows the CIS coefficients of the $1^{1} \mathrm{~A}_{2}$ state obtained using various methods with different active spaces or basis sets. In the case of KS-CIS, the $2 b_{2} \rightarrow 2 b_{1}$ excitation is dominant and thus their excitation energies are not only accurate, but also insensitive to the active space size, since both small and large active spaces include the $2 b_{2}$ and $2 b_{1}$ orbitals. In contrast, HF-CIS for both grid and
Gaussian basis sets needs more configurations in addition to the $2 b_{2} \rightarrow 2 b_{1}$ excitation. In fact, as the active space or basis set size increases, the coefficient corresponding to the $2 b_{2} \rightarrow 2 b_{1}$ excitation becomes considerably small, while the contribution from $2 b_{2} \rightarrow 3 b_{1}$ or $2 b_{2} \rightarrow 4 b_{1}$ becomes more dominant.

Table 3 shows those orbitals involved in the excitation to the $1^{1} \mathrm{~A}_{2}$ state. The highest occupied molecular orbitals (HOMOs) denoted as $2 b_{2}$ from KLI and HF have very similar shapes at least to the naked eye. In contrast, as stressed above, their virtual orbitals are substantially different in shape and size. In particular, the $2 b_{1}$ orbital from KLI is more compact than those from HF. Moreover, the energy gap (4.19 eV) between $2 b_{2}$ and $2 b_{1}$ from KLI is much smaller, that is closer to the best estimation value $(3.88 \mathrm{eV})$, than those from HF (13.17 or $13.64 \mathrm{eV}$ ). As a result, HF-CIS needs to add more virtual orbitals with the same symmetry to improve accuracy (Fig. 4). In general, KLI gives a number of bound virtual orbitals with negative energies and compact shapes, whereas HF produces unbound virtual orbitals with positive energies and diffuse shapes. ${ }^{29}$ Thus, the excitation to the $1^{1} \mathrm{~B}_{1}$ and $2{ }^{1} \mathrm{~A}_{1}$ states also shows similar trends as depicted in Fig. S3 (ESI $\dagger$ ).

Benzene. Unlike formaldehyde, MCs are vital to properly express the excited states of benzene that involve various $\pi \rightarrow \pi^{*}$ excitations. In Table 2 , the $1^{1} \mathrm{~B}_{1 \mathrm{u}}$ and $1^{1} \mathrm{~B}_{2 \mathrm{u}}$ states correspond to the excitations from two degenerate $\pi$ bonding orbitals to

Table 3 Orbitals of formaldehyde calculated using $\mathrm{KLI}$ and $\mathrm{HF}^{a}$

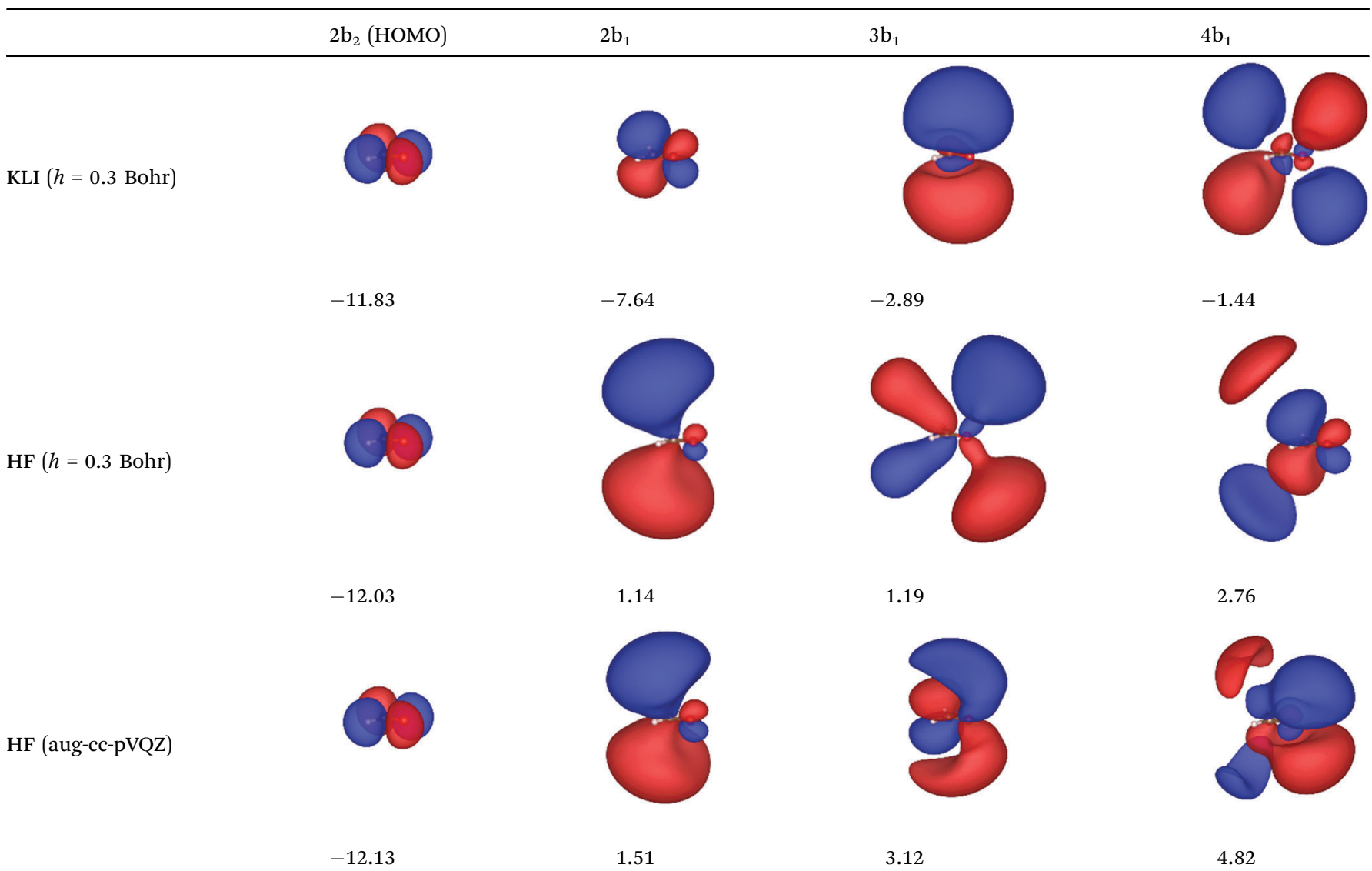

${ }^{a}$ The values below each orbital figure indicate corresponding orbital energies (unit: eV). 


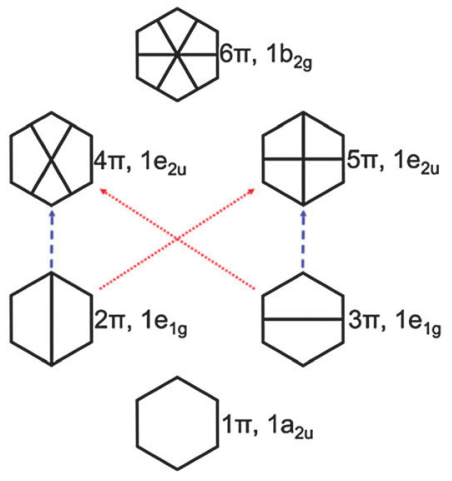

Fig. $5 \pi$ orbital diagram of benzene. The red and blue dashed arrows indicate the excitations to the $1^{1} \mathrm{~B}_{2 u}$ and $1^{1} \mathrm{~B}_{2 u}$ states, respectively (see also Fig. S4, ESI†).

two degenerate $\pi$ antibonding orbitals as schematically depicted in Fig. 5. Because degenerate orbitals have the same symmetry representation, (e.g., $1 \mathrm{e}_{1 \mathrm{~g}}$ for $\pi$ bonding orbitals of benzene), MC nature of such excited states is unavoidable. For notations, we denote $\pi$ orbitals formed by the $2 \mathrm{p}$ and $3 \mathrm{p}$ carbon atomic orbitals as $n \pi$ and $n^{\prime} \pi$, respectively ( $c f$. Fig. 5-7).

In the case of KS-CIS, the $1^{1} \mathrm{~B}_{1 \mathrm{u}}$ state is represented by mainly two $\pi \rightarrow \pi^{*}$ excitations as shown in Fig. $6: 2 \pi \rightarrow 5 \pi$ and $3 \pi \rightarrow 4 \pi$. In constrast, HF-CIS (grid) has no such excitation characteristics within the small active spaces (" 3 " and " 6 "), leading to the large deviations in the excitation energies (Table 2). For the active space " 45 ", more configurations especially involving $\mathrm{n} \pi \rightarrow \mathrm{n}^{\prime} \pi$ excitations in addition to the two $\pi \rightarrow \pi^{*}$ excitations contribute to the corresponding excitation energy, and then the excitation energy of HF-CIS (grid) is comparable to that of KS-CIS. This indicates that for HF-CIS (grid) the two configurations are not sufficient to achieve a similar accuracy to KLI-CIS and thus more configurations should be added. A similar trend is observed for the Gaussian basis sets.

Fig. 7 shows the orbitals involved in the excitation to the $1^{1} B_{1 u}$ state. Like the case of formaldehyde, KLI and HF have similar occupied $\pi$ orbitals $(2 \pi, 3 \pi)$, but considerably different

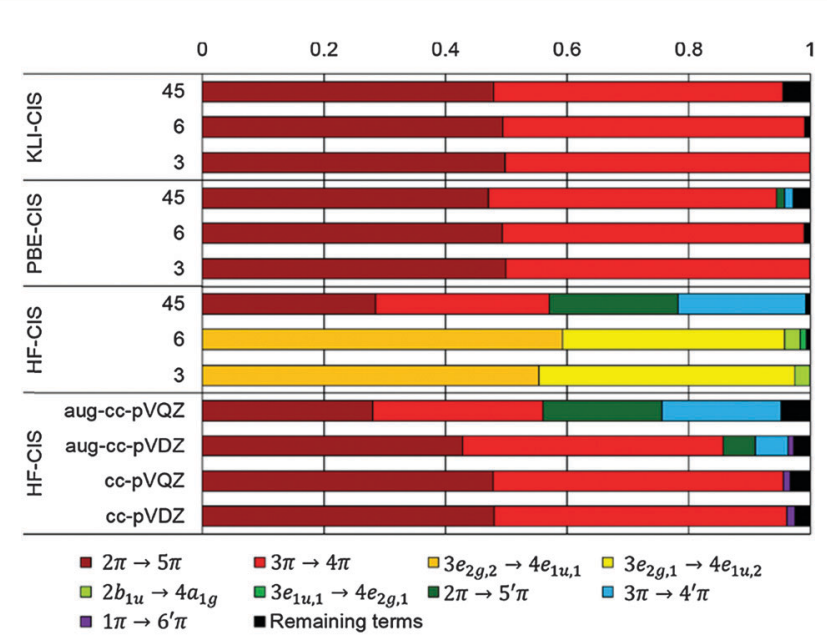

Fig. $6 \mathrm{CIS}$ coefficients for the $1^{1} \mathrm{~B}_{1 \mathrm{u}}$ state of benzene.

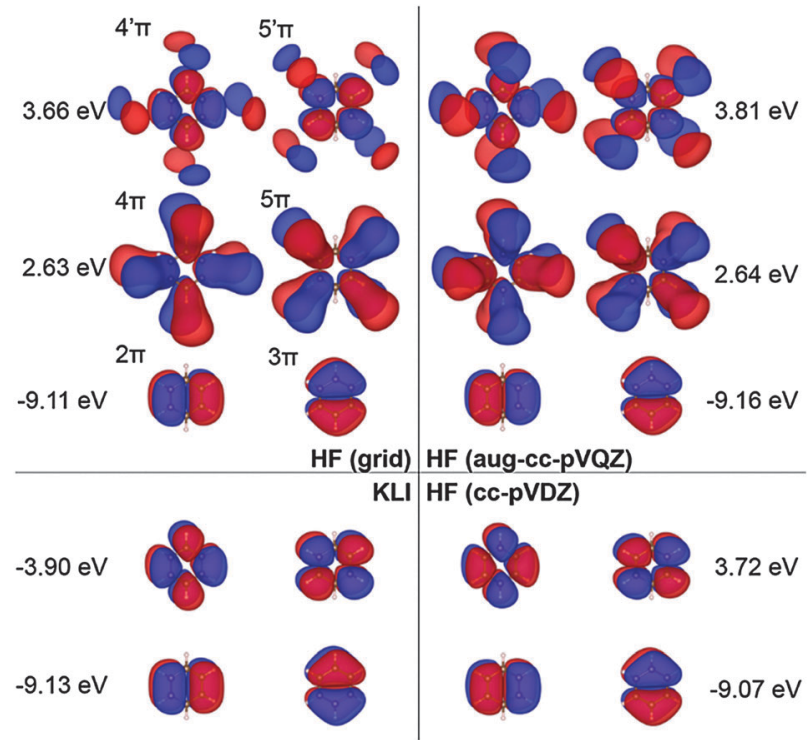

Fig. 7 Selected $\pi$ molecular orbitals and corresponding energies of benzene calculated using $\mathrm{KLI}$ and $\mathrm{HF}$.

virtual orbitals with strong dependence on the basis set size. The relatively small Gaussian basis set (cc-pVDZ) gives compact $4 \pi$ and $5 \pi$ orbitals that are similar to those from KLI. However, HF with a larger basis set such as aug-cc-pVQZ or numerical grid yields much more diffuse orbitals. KLI $(5.23 \mathrm{eV})$ has smaller energy gaps between $(2 \pi, 3 \pi)$ and $(4 \pi, 5 \pi)$ than HF (grid: $11.74 \mathrm{eV}$ and aug-cc-pVQZ: $11.8 \mathrm{eV}$ ). Therefore, HF-CIS ("45" and aug-cc-pVQZ) compensates the large energy gap by adding the contribution from the $2 \pi \rightarrow 5^{\prime} \pi$ and $3 \pi \rightarrow 4^{\prime} \pi$ excitations to the $2 \pi \rightarrow 5 \pi$ and $3 \pi \rightarrow 4 \pi$ excitations.

Formamide. As observed from the above two examples, KLICIS needs a single configuration for non-degenerate excitations and MCs for degenerate excitations, whereas HF-CIS mostly requires MCs for any excitation. PBE-CIS shows similar trends with KLI-CIS. For the excitation of formamide to the $1^{1} \mathrm{~A}^{\prime \prime}$ state, a single configuration involving $\left(10 \mathrm{a}^{\prime} \rightarrow 3 \mathrm{a}^{\prime \prime}\right)$ is dominant for KLI-CIS (Fig. S5, ESI $\dagger$ ). However, the $2^{1} \mathrm{~A}^{\prime}$ and $3^{1} \mathrm{~A}^{\prime}$ states demand MCs even for KLI-CIS, though the two states are not due to degenerate excitations as shown in Fig. 8 (also see Fig. S5, $\mathrm{ESI} \dagger)$. Moreover, the excitation energies of formamide computed from all methods have relatively larger deviations from the best estimation values compared to the other molecules. For instance, the excitation energy of $1^{1} \mathrm{~A}$ " from KLI-CIS converged within $0.02 \mathrm{eV}$ with respect to the active space size but yet has a large deviation $(0.83 \mathrm{eV})$. This may be attributed to its intrinsic multiple-excitation characteristics. In this case, we expect that the large deviation can be reduced by including multiple excitations in the CI expansion. Nonetheless, KLI-CIS still gives better excitation energies with respect to the best estimation values than PBE- and HF-CIS.

Hydrogen molecule. A hydrogen molecule is the simplest neutral molecule. It has a well-defined symmetry and only two electrons. Therefore, as expected, all methods show similar good accuracy (Fig. S6, ESI $\dagger$ ). For example, the excitation energies of 


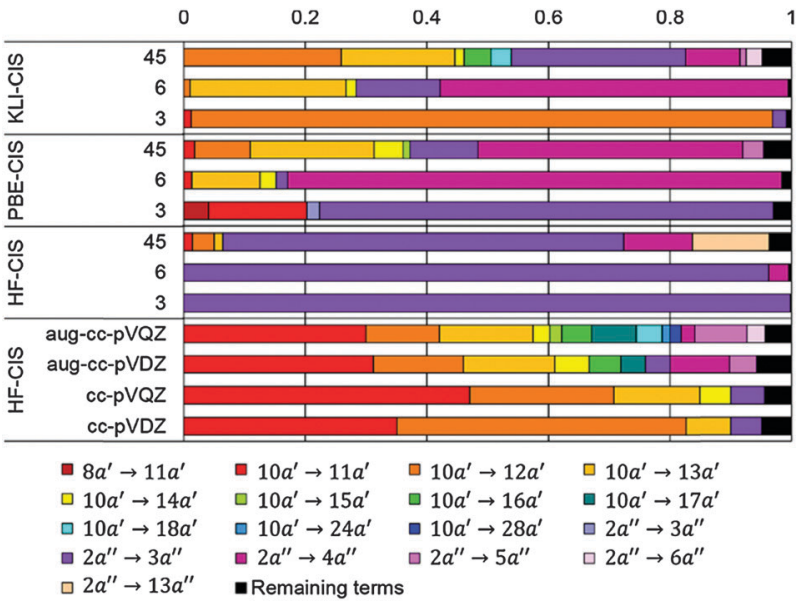

Fig. $8 \mathrm{CIS}$ coefficients for the $3^{1} \mathrm{~A}^{\prime}$ state of formamide.

HF-CIS (grid) with the active space " 45 " are comparable to the KLI- or PBE-CIS results, although they are still sensitive to the size of active space (Table 2). Through the expansion of the active space from " 6 " to " 45 ", the excitation energies of HF-CIS (grid) were lowered by $0.4-0.79 \mathrm{eV}$, while those of KLI-CIS were varied within $0.06-0.09 \mathrm{eV}$.

\section{Conclusions}

Real-space numerical grid methods are promising for accurate electronic structure calculations of large molecules, because their computational costs can be reduced through massive parallelization as well as linear scaling algorithms and their accuracy can be systematically improved toward complete basis set limits. In particular, those methods are inherently good for excited state calculations in which large basis sets including diffuse functions are essential. We developed a CIS method using Lagrange-sinc functions localized on a uniform grid. The nonlocal HF exchange operator reduces the sparsity of the Hamiltonian matrix, giving rise to high computational costs. Therefore, the traditional HF-based CIS seems impractical for grid-based methods. Instead, we proposed a KS-based CIS in particular with an exact exchange potential. KLI and PBE orbitals as well as HF orbitals were used to build a reference configuration and their relative accuracy for excitation energies of molecules has been assessed.

Since the grid-based method uses an iterative diagonalization scheme, the size of active space should be determined in a way to compromise between computational costs and accuracy. For our test molecules with the active space using all occupied orbitals and 45 virtual orbitals, KLI-CIS shows smallest deviations (MAD = $0.44 \mathrm{eV}$ ) in excitation energy calculations with respect to the known best estimation values, followed by PBE-CIS (MAD $=0.55 \mathrm{eV}$ ), while HF-CIS (MAD $=0.91 \mathrm{eV}$ ) causes significantly large deviations. We also found that excitation energies from KLI-CIS are less sensitive to the size of active space, whereas the results from HF-CIS are very sensitive. Such differences originate from the unique features of KLI orbitals; HF-like occupied orbitals, but KS-like virtual orbitals. Compared to HF, KLI yields many bound virtual orbitals with more compact shapes and smaller energy gaps between occupied and virtual orbitals. As a result, for $\mathrm{H}_{2}$, benzene, and formaldehyde, KLICIS needs mostly a specific single configuration for non-degenerate excitations and multiconfigurations for degenerate excitations, whereas HF-CIS demands multiconfigurations for any excitation. However, for formamide, both KLI-CIS and HF-CIS require multiconfigurations to obtain accurate excitation energies which may be due to its multiple-excitation character. Consequently, the size of active space should be cautiously determined as a convergence parameter especially for HF-CIS.

The size of active space is directly related to computational costs of multiconfiguration methods. For CI singles and doubles (CISD) calculations, the number of configurations is approximately proportional to $N_{\text {occ }}{ }^{2} N_{\text {vir }}{ }^{2}$. Based on the CIS results in this work, we expect that KLI-CISD should be more cost-effective because it needs smaller active space to achieve similar accuracy than HFCISD. For benzene as an example, KLI-CISD with the active space "45" and "6" have 315 and 2644 times smaller number of configurations than HF-CISD with aug-cc-pVQZ, respectively ( $c f$. Table 1). Hence, we believe that KLI-based multiconfiguration methods combined with numerical grid basis sets will provide a new promising way for accurate electronic structure calculations of large systems.

\section{Acknowledgements}

This work was supported by Basic Science Research Programs (NRF-2012R1A1A1004154), the EDISON Program (NRF-2012 M3C1A6035359), and the MIR center (No. 20090083525) through the NRF funded by the Korea government [MSIP]. The authors would like to acknowledge the support from KISTI supercomputing center for computing time (KSC-2013-C1-028). W.Y.K. is also grateful for financial support from Chung-Am Fellowship and EWon assistant professorship.

\section{Notes and references}

1 I. Samish, C. M. MacDermaid, J. M. Perez-Aguilar and J. G. Saven, Annu. Rev. Phys. Chem., 2011, 62, 129-149.

2 J. K. Nørskov, T. Bligaard, J. Rossmeisl and C. H. Christensen, Nat. Chem., 2009, 1, 37-46.

3 S. Martí, J. Andrés, V. Moliner, E. Silla, I. Tuñón and J. Bertrán, Chem. Soc. Rev., 2008, 37, 2634-2643.

4 L. Wang, G. Nan, X. Yang, Q. Peng, Q. Li and Z. Shuai, Chem. Soc. Rev., 2010, 39, 423-434.

5 V. Nanda and R. L. Koder, Nat. Chem., 2010, 2, 15-24.

6 N. Lambert, Y.-N. Chen, Y.-C. Cheng, C.-M. Li, G.-Y. Chen and F. Nori, Nat. Phys., 2013, 9, 10.

7 X. Andrade, J. Alberdi-Rodriguez, D. A. Strubbe, M. J. T. Oliveira, F. Nogueira, A. Castro, J. Muguerza, A. Arruabarrena, S. G. Louie, A. Aspuru-Guzik, A. Rubio and M. A. L. Marques, J. Phys.: Condens. Matter, 2012, 24, 233202.

8 J. Enkovaara, C. Rostgaard, J. J. Mortensen, J. Chen, M. Dułak, L. Ferrighi, J. Gavnholt, C. Glinsvad, V. Haikola, H. A. Hansen, H. H. Kristoffersen, M. Kuisma, A. H. Larsen, 
L. Lehtovaara, M. Ljungberg, O. Lopez-Acevedo, P. G. Moses, J. Ojanen, T. Olsen, V. Petzold, N. A. Romero, J. StausholmMøller, M. Strange, G. A. Tritsaris, M. Vanin, M. Walter, B. Hammer, H. Häkkinen, G. K. H. Madsen, R. M. Nieminen, J. K. Nørskov, M. Puska, T. T. Rantala, J. Schiøtz, K. S. Thygesen and K. W. Jacobsen, J. Phys.: Condens. Matter, 2010, 22, 253202.

9 S. Mohr, L. E. Ratcliff, P. Boulanger, L. Genovese, D. Caliste, T. Deutsch and S. Goedecker, J. Chem. Phys., 2014, 140, 204110.

10 C. K. Skylaris, P. D. Haynes, A. A. Mostofi and M. C. Payne, J. Chem. Phys., 2005, 122, 084119.

11 J. I. Iwata, D. Takahashi, A. Oshiyama, T. Boku, K. Shiraishi, S. Okada and K. Yabana, J. Comput. Phys., 2010, 229, 2339-2363.

12 L. Kronik, A. Makmal, M. L. Tiago, M. M. G. Alemany, M. Jain, X. Huang, Y. Saad and J. R. Chelikowsky, Phys. Status Solidi, 2006, 243, 1063-1079.

13 F. Bottin, S. Leroux, A. Knyazev and G. Zérah, Comput. Mater. Sci., 2008, 42, 329-336.

14 E. Di Napoli and M. Berljafa, Comput. Phys. Commun., 2013, 184, 2478-2488.

15 Y. Zhou and Y. Saad, Numer. Algorithm., 2008, 47, 341-359. 16 A. Sitt, L. Kronik, S. Ismail-Beigi and J. R. Chelikowsky, Phys. Rev. A: At., Mol., Opt. Phys., 2007, 76, 054501.

17 C. J. Cramer and D. G. Truhlar, Phys. Chem. Chem. Phys., 2009, 11, 10757-10816.

18 A. J. Cohen, P. Mori-Sanchez and W. Yang, Chem. Rev., 2012, 112, 289-320.

19 J. B. Krieger, Y. Li and G. J. Iafrate, Phys. Rev. A: At., Mol., Opt. Phys., 1992, 45, 101.

20 J. B. Krieger, Y. Li and G. J. Iafrate, Phys. Rev. A: At., Mol., Opt. Phys., 1992, 46, 5453-5458.

21 J. B. Krieger, Y. Li and G. J. Iafrate, Phys. Lett. A, 1990, 148, 470-474.

22 J. B. Krieger, Y. Li and G. J. Iafrate, in Density Functional Theory, ed. E. K. U. Gross and R. M. Dreizler, Springer US, 1995, pp. 191-216.

23 G. J. Iafrate and J. B. Krieger, J. Chem. Phys., 2013, 138, 094104.

24 F. Della Sala and A. Görling, J. Chem. Phys., 2001, 115, 5718-5732.

25 F. Della Sala and A. Görling, J. Chem. Phys., 2002, 116, 5374-5388.

26 F. Della Sala and A. Görling, J. Chem. Phys., 2003, 118, 10439-10454.

27 O. Gritsenko and E. J. Baerends, Phys. Rev. A: At., Mol., Opt. Phys., 2001, 64, 042506.

28 M. Grüning, O. V. Gritsenko and E. J. Baerends, J. Chem. Phys., 2002, 116, 6435-6442.

29 J. Kim, K. Hong, S. Choi and W. Y. Kim, Bull. Korean Chem. Soc., 2015, 36, 998-1007.

30 R. van Meer, O. V. Gritsenko and E. J. Baerends, J. Chem. Theory Comput., 2014, 10, 4432-4441.

31 E. J. Baerends, O. V Gritsenko and R. van Meer, Phys. Chem. Chem. Phys., 2013, 15, 16408-16425.

32 Y.-H. Kim, M. Stadele and R. M. Martin, Phys. Rev. A: At., Mol., Opt. Phys., 1999, 60, 3633-3640.

33 S. Grimme, Chem. Phys. Lett., 1996, 259, 128-137.

34 S. Grimme and M. Waletzke, J. Chem. Phys., 1999, 111, 5645.

35 P. Bour, Chem. Phys. Lett., 2001, 345, 331-337.

36 L. Veseth, J. Chem. Phys., 2001, 114, 8789.
37 T. Hupp, B. Engels, F. Della and G. Andreas, Chem. Phys. Lett., 2002, 360, 175-181.

38 T. Hupp, B. Engels and A. Görling, J. Chem. Phys., 2003, 119, 11591.

39 M. Roemelt, D. Maganas, S. DeBeer and F. Neese, J. Chem. Phys., 2013, 138, 204101.

40 C. Gutlé, J. L. Heully, J. B. Krieger and A. Savin, Phys. Rev. A: At., Mol., Opt. Phys., 2002, 66, 012504.

41 B. Kaduk, T. Kowalczyk and T. Van Voorhis, Chem. Rev., 2012, 112, 321-370.

42 T. Yanai, R. J. Harrison and N. C. Handy, Mol. Phys., 2005, 103, 413-424.

43 S. Choi, K. Hong, J. Kim and W. Y. Kim, J. Chem. Phys., 2015, 142, 094116.

44 D. Baye, Phys. Status Solidi, 2006, 243, 1095-1109.

45 K. Varga and S. T. Pantelides, Phys. Status Solidi, 2006, 243, 1110-1120.

46 L. Genovese, T. Deutsch, A. Neelov, S. Goedecker and G. Beylkin, J. Chem. Phys., 2006, 125, 074105.

47 S. A. Losilla, D. Sundholm and J. Jusélius, J. Chem. Phys., 2010, 132, 024102.

48 J. Jusélius and D. Sundholm, J. Chem. Phys., 2007, 126, 094101.

49 H.-S. Lee and M. E. Tuckerman, J. Chem. Phys., 2008, 129, 224108.

50 G. Beylkin and L. Monzón, Appl. Comput. Harmon. Anal., 2005, 19, 17-48.

51 M. M. Mehine, S. A. Losilla and D. Sundholm, Mol. Phys., 2013, 111, 2536.

52 M. Schreiber, M. R. Silva-Junior, S. P. A. Sauer and W. Thiel, J. Chem. Phys., 2008, 128, 134110.

53 M. R. Silva-Junior, M. Schreiber, S. P. A. Sauer and W. Thiel, J. Chem. Phys., 2008, 129, 104103.

54 J. P. Perdew, K. Burke and M. Ernzerhof, Phys. Rev. Lett., 1996, 77, 3865-3868.

55 THEOS, http://theossrv1.epfl.ch/Main/Pseudopotentials, accessed Jan. 19, 2015.

56 M. J. Frisch, G. W. Trucks, H. B. Schlegel, G. E. Scuseria, M. A. Robb, J. R. Cheeseman, G. Scalmani, V. Barone, B. Mennucci, G. A. Petersson, H. Nakatsuji, M. Caricato, X. Li, H. P. Hratchian, A. F. Izmaylov, J. Bloino, G. Zheng, J. L. Sonnenberg, M. Hada, M. Ehara, K. Toyota, R. Fukuda, J. Hasegawa, M. Ishida, T. Nakajima, Y. Honda, O. Kitao, H. Nakai, T. Vreven, J. A. Montgomery, Jr., J. E. Peralta, F. Ogliaro, M. Bearpark, J. J. Heyd, E. Brothers, K. N. Kudin, V. N. Staroverov, R. Kobayashi, J. Normand, K. Raghavachari, A. Rendell, J. C. Burant, S. S. Iyengar, J. Tomasi, M. Cossi, N. Rega, J. M. Millam, M. Klene, J. E. Knox, J. B. Cross, V. Bakken, C. Adamo, J. Jaramillo, R. Gomperts, R. E. Stratmann, O. Yazyev, A. J. Austin, R. Cammi, C. Pomelli, J. W. Ochterski, R. L. Martin, K. Morokuma, V. G. Zakrzewski, G. A. Voth, P. Salvador, J. J. Dannenberg, S. Dapprich, A. D. Daniels, O. Farkas, J. B. Foresman, J. V. Ortiz, J. Cioslowski and D. J. Fox, Gaussian 09, Revision A.02, Gaussian, Inc., Wallingford CT, 2009.

57 W. Kolos and L. Wolniewicz, J. Chem. Phys., 1965, 43, 2429-2441. 58 W. Kolos and L. Wolniewicz, J. Chem. Phys., 1969, 50, 3228. 59 L. Wolniewicz and K. Dressler, J. Chem. Phys., 1988, 88, 3861. 